\title{
BMJ Open Behaviour change interventions for the management of Raynaud's phenomenon: a systematic literature review
}

\author{
Jo Daniels, ${ }^{1}$ John D Pauling, ${ }^{2,3}$ Christopher Eccleston ${ }^{4,5}$
}

To cite: Daniels J, Pauling JD, Eccleston C. Behaviour change interventions for the management of Raynaud's phenomenon: a systematic literature review. BMJ Open 2018;8:e024528. doi:10.1136/ bmjopen-2018-024528

- Prepublication history and additional material for this paper are available online. To view these files, please visit the journal online (http://dx.doi. org/10.1136/bmjopen-2018024528).

Received 30 May 2018 Revised 6 October 2018 Accepted 2 November 2018

Check for updates

(C) Author(s) (or their employer(s)) 2018. Re-use permitted under CC BY-NC. No commercial re-use. See rights and permissions. Published by BMJ.

${ }^{1}$ Department of Psychology, The University of Bath, Bath, UK

2Department of Rheumatology, Royal National Hospital for Rheumatic Diseases, Bath, UK ${ }^{3}$ Department of Pharmacy and Pharmacology, The University of Bath, Bath, UK

${ }^{4}$ Centre for Pain Research, University of Bath, Bath, UK ${ }^{5}$ Department of Clinical and Health Psychology, Ghent University, Ghent, Belgium

Correspondence to

Dr Jo Daniels;

j.daniels@bath.ac.uk

\section{ABSTRACT}

Objectives Raynaud's phenomenon (RP) is a significant cause of morbidity. Vasodilator medications cause unwanted adverse effects, with behavioural and lifestyle changes forming the mainstay of self-management; this is difficult to implement successfully. The objectives of this study were to evaluate the efficacy of behaviour change interventions for RP and identify learning points for future treatment development.

Design Systematic literature review and narrative synthesis of findings.

Data sources EMBASE, MEDLINE, Cochrane and PsycINF0 were searched for eligible studies on 22 August 2017.

Eligibility criteria Randomised controlled trials (RCTs) of behaviour change interventions with at least one control comparator arm.

Data extraction and synthesis Study selection, data extraction and risk of bias were assessed independently by two reviewers, reaching consensus with a third when necessary. Primary outcomes of interest included severity/ impact, frequency and duration of RP episodes, pain, disability, adverse events and study withdrawal.

Results of 638 articles retrieved, eight studies fulfilled criteria for inclusion. Biofeedback was the active behaviour change treatment arm for seven studies, with one study reporting a behavioural intervention. Studies were published 1978-2002; six were USA-based studies, one German and one Swedish. Using Cochrane Risk of Bias assessment, studies were assessed to be overall at high risk of bias, with the exception of one large RCT. The total sample included 495 participants (study median=29), with a median age of 39.5 years and preponderance towards females (73\%). Five studies reported significant effects in primary outcomes of interest; however, due to missing data, relative efficacy of interventions could not be reliably assessed.

Conclusions There is no evidence to support or refute claims of the efficacy of behaviour change interventions for the management of RP. There remains a strong case for developing and testing behaviour change interventions that focus on self-management; however, theoretical development and advancement in trial quality is imperative to underpin future work.

PROSPERO registration number CRD42017049643.

\section{INTRODUCTION}

Peripheral vasoconstriction of thermoregulatory precapillary arterioles and arteriovenous anastomoses is a normal physiological

\section{Strengths and limitations of this study}

This is the first systematic literature review of behaviour change interventions in Raynaud's phenomenon, making a novel contribution to the field of clinical rheumatology.

- The published protocol was strictly adhered to, reducing likelihood of bias and offering a robust systematic review methodology to satisfy study objectives.

- Eligibility criteria were restricted to randomised controlled trials of behaviour change interventions in order to meet study objectives, resulting in pool of eight included studies without language or publication date restrictions.

Due to limitations in study design and incompleteness of data, a meta-analysis to assess comparative efficacy was not viable; a narrative synthesis of study findings is reported.

thermoregulatory response to cold exposure. ${ }^{1}$ Raynaud's phenomenon (RP) describes excessive peripheral vasoconstriction to cold exposure and/or emotional stress. Attacks of $\mathrm{RP}$ are associated with characteristic digital colour changes (reflecting blood oxygenation and tissue perfusion). ${ }^{2}{ }^{3}$ Tissue ischaemia (and subsequent reperfusion) during attacks of RP results in pain and paraesthesia causing distress, loss of hand function and reduced quality of life. ${ }^{23}$ RP is common, affecting approximately $5 \%$ of people. The majority of sufferers have a functional vasospastic disorder that, while intrusive, is otherwise benign in nature (termed primary RP). Digital perfusion is generally normal in between attacks. The term secondary RP is applied to disorders in which RP symptoms occur as a result of disturbed digital tissue perfusion related to separate underlying pathology. Important causes of secondary RP are autoimmune rheumatic diseases such as systemic sclerosis ( $\mathrm{SSc}$ ), in which a progressive obliterative microangiopathy can result in persistent digital ischaemia and tissue damage. ${ }^{4}$ Despite being rare, with an 
estimated prevalence of 250/million, SSc is often used as the focus of RP research. ${ }^{5}$

Cold exposure appears to be the major factor precipitating RP symptoms in SSc, although emotional stress provokes symptoms in approximately $30 \%$ of episodes. ${ }^{6}$ Emotional stress appears to represent a more prominent aggravating factor in primary $\mathrm{RP}^{7}$ Thematically relevant emotional stressors appear to be important. For example, imagining the threat of cold exposure (losing gloves and car keys during a snowstorm) results in reduced finger temperature in people with primary RP but not healthy controls. ${ }^{8}$ These observations could have important implications for the behavioural management of RP within different patient populations. Self-management measures are typically included in recommendations on the management of RP but do not typically extend beyond general advice on avoiding cold exposure, conserving heat loss, smoking cessation, increasing exercise and reducing stress levels. ${ }^{9}{ }^{10}$ Adherence with interventions of this nature is typically poor, with estimates of $30 \%-50 \%$ of patients demonstrating poor compliance, regardless of condition, expected outcome or setting. ${ }^{11}$ Despite the perceived importance of non-pharmacological interventions for RP, the comparative efficacy, adoption and compliance with lifestyle interventions has not been fully evaluated. A number of behaviour change interventions have been tested for RP, but the comparative efficacy of a range of interventions within different disease populations (primary and secondary RP) has not previously formed the focus of a systematic review. We report the findings of the first systematic literature review to evaluate the efficacy of behaviour change interventions for the management of primary and secondary RP. We consider how the findings inform recommendations on behaviour change interventions for RP and future research efforts in this field.

\section{Objectives}

The specific objectives of this systematic review were to: (1) assess the comparative safety and efficacy of a range of behavioural interventions for the management of symptoms associated with primary and secondary RP and (2) identify what we can learn from the studies reviewed to inform study protocols for future behaviour change interventions for RP.

\section{METHODS}

The protocol and supplementary material used to develop this systematic review has been published with open access ${ }^{12}$ and registered in the International Prospective Register of Systematic Reviews and available from http://bmjopen.bmj.com/content/7/8/e017039. This systematic review complies with the Preferred Reporting Items for Systematic Review and Meta-Analysis Protocols (PRISMA) guidelines (see online supplementary materials, table 1).

\section{Search strategy}

The search strategy was designed to identify treatment studies examining the efficacy of behaviour change interventions in the treatment of adults with RP (primary or secondary). MEDLINE, EMBASE, PsycINFO and the Cochrane library were searched on 22 August 2017 using terms developed by the research team and in collaboration with an information specialist $(\mathrm{JH})$. The term 'behaviour change intervention' was defined a priori as interventions that target symptomatic relief of RP through directed or advised change in patient-determined behaviour ${ }^{12}$ and interventions designed to encourage sustained change in patient behaviour that directly and purposefully improves either physical or psychological well-being. To include all possible permutations of the interventions designed to change behaviour, the search terms were purposively broad and inclusive (behavio $(\mathrm{u})$ ral therapy, cognitive therapy, education, psychoeducation, biofeedback, clinical psychology, psychotherapy, self-management, cognitive behavio $(u) r$ therapy and behavioural medicine). See online supplementary tables 2 and 3 for further detail.

To ensure a comprehensive capture of relevant and high-quality studies, reference lists of articles included at the full-text review stage were hand-searched on 24 November 2017. Authors of papers in the final stage of the review were also contacted for further grey literature. Published studies in any language were included, with no date restrictions.

\section{Inclusion criteria}

Randomised controlled trials (RCTs) testing behaviour change interventions for the treatment or management of adults with RP (primary or secondary) were included in the systematic review. Due to the lack of consensus in the use of RP diagnostic criteria, all clinical definitions were included. Other clinical trial designs (eg, non-RCTs and those without a control comparator) were excluded; however non-blinded studies were included due to the difficulties of blinding in trials of this nature. Duration of intervention was not subject to a minimum criteria, providing the study within which the intervention was assessed fully met all inclusion criteria.

\section{Selection of studies}

Studies generated by the initial search were screened by two authors for eligibility (JD and JP). All full texts were reviewed and independently rated for inclusion by two review authors using a prespecified, published data extraction form. ${ }^{12}$ Bibliographies of included studies and grey literature search were conducted by the first author and were subject to review of eligibility. Discrepancies at each stage were resolved through consultation with the third author reviewer (CE).

\section{Data extraction}

Data were independently extracted from included studies by two review authors, using the prespecified 
data extraction form (online supplementary materials, table 4). Study authors were contacted in cases of missing or incomplete data. In addition to outcome data, data pertaining to the quality of psychotherapeutic interventions were extracted, specifically reference to a theoretical model, level of therapist training and whether the integrity of the intervention was checked. These criteria were drawn from an authoritative review of empirically supported psychotherapies. ${ }^{13}$

Due to the absence of agreement regarding domains of measurement or measurement technology in $\mathrm{RP},{ }^{14}$ the primary outcome measures chosen for our analyses mirror those adopted in a recent generic systematic review protocol for $\mathrm{RP},{ }^{15}$ including: (1) severity/impact of $R P$ episodes assessed using visual analogue scales (VAS); Likert scales or the Raynaud's Condition Score (RCS) ${ }^{16}$ (either at a single time point using a standardised recall period) or as an average daily score (obtained from the RCS diary or equivalent from RP symptom diary); (2) frequency of RP attacks (adopted from the RCS diary or equivalent symptom diary approach) reported as average daily or weekly frequency of RP attacks; (3) duration of $R P$ attacks (adopted from the RCS diary or equivalent symptom diary approach) reported as the average daily duration of RP attacks over 1-2 weeks; (4) pain assessed using a VAS or Likert scale (reporting intensity of pain during RP attacks); and (5) patient assessment of disability due to RP/interference on daily activities, for example, the Scleroderma Health Assessment Questionnaire (SHAQ), ${ }^{17}$ RP VAS or equivalent; (6) adverse events (hospitalisation/death); and (7) withdrawals from study were also included within primary outcomes.

Secondary outcomes included: (1) physician global assessment of severity/impact of RP, (2) patient global assessment of function/disability secondary to RP (eg, the SHAQ score); (3) change in digital ulceration (positive/negative); (4) treatment preference; and (5) general improvement (self-reported overall improvement). Most RP clinical trials involve assessments over one or more weeks. Sensitivity analyses were planned for trials with marked differences in durations of treatment/assessment.

\section{Risk of bias}

Risk of bias was independently assessed on an outcome and study level by two authors using the Cochrane risk of bias assessment tool (online supplementary materials, table 5). ${ }^{18}$ Unresolved discrepancies were reviewed by the third author. Risk of bias was assessed according to the following dimensions: random sequence generation (adequate description and method of participant allocation in accordance with standard randomisation); allocation concealment (adequate concealment of group assignment to prevent selection bias); blinding of participants and personnel (adequacy of measures taken to prevent performance bias and conceal group assignment); blinding outcome assessors (adequacy of measures taken to prevent detection bias and conceal group assignment to outcome assessors); incomplete data (adequacy of the management of missing data and potential implications for bias); selective outcome reporting (reporting bias relating to the consistency between prespecified and reported outcomes); and other sources of bias (other concerns not covered elsewhere but may lead to a risk of bias). Eligible studies were rated as high, low or unclear (risk of bias) on each of these dimensions, culminating in an overall risk of bias (high/low/unclear) in accordance with the Cochrane handbook for systematic reviews of interventions. ${ }^{18}$ Investigator agreement was evaluated using Kappa statistics.

\section{Quality of evidence}

Quality of evidence and confidence in estimates of effect were assessed using Grading of Recommendations, Assesment, Development and Evaluation (GRADE): (in) consistency of effect, imprecision, indirectness and publication bias. ${ }^{19}$ A GRADE summary of findings table was planned on this basis.

\section{Data synthesis and analysis}

A comprehensive meta-analysis and secondary subgroup analysis were planned; however, due to the low numbers of studies included for analysis, insufficient data available from several studies and the heterogeneity of the available data and outcomes, an attempt at formal meta-analysis was not considered clinically or statistically meaningful. Funnel and forest plots were not generated for the aforementioned reasons.

In the absence of meta-analyses, a narrative (descriptive) analysis of primary and secondary outcomes was planned to include: description of individual study outcomes in terms of frequency/severity/duration of episodes, patient global assessment of disability and changes on RCS scores where available; analysis of reported design or intervention features in behaviour change interventions; and analysis of reported considerations as regards of future behaviour-change interventions. ${ }^{12}$

\section{Patient and public involvement statement}

Patients and/or public were not directly involved in the conduct of this study; however, this study forms part of a broader programme of research that is supported by a patient advisory group.

\section{RESULTS}

\section{Selection and inclusion of studies}

The initial search generated 638 studies, resulting in 304 abstracts/titles following removal of duplicates. Independent review at this stage removed a further 282 for irrelevance, with 22 studies retrieved for full-text retrieval. Full-text review resulted in removal of a further 14 studies (see figure 1), leaving a final set of eight studies reported across nine papers. This included a single original study associated with a follow-up paper retrieved from the authors for inclusion; the late addition was attributed to the original paper having been written in Swedish, and 


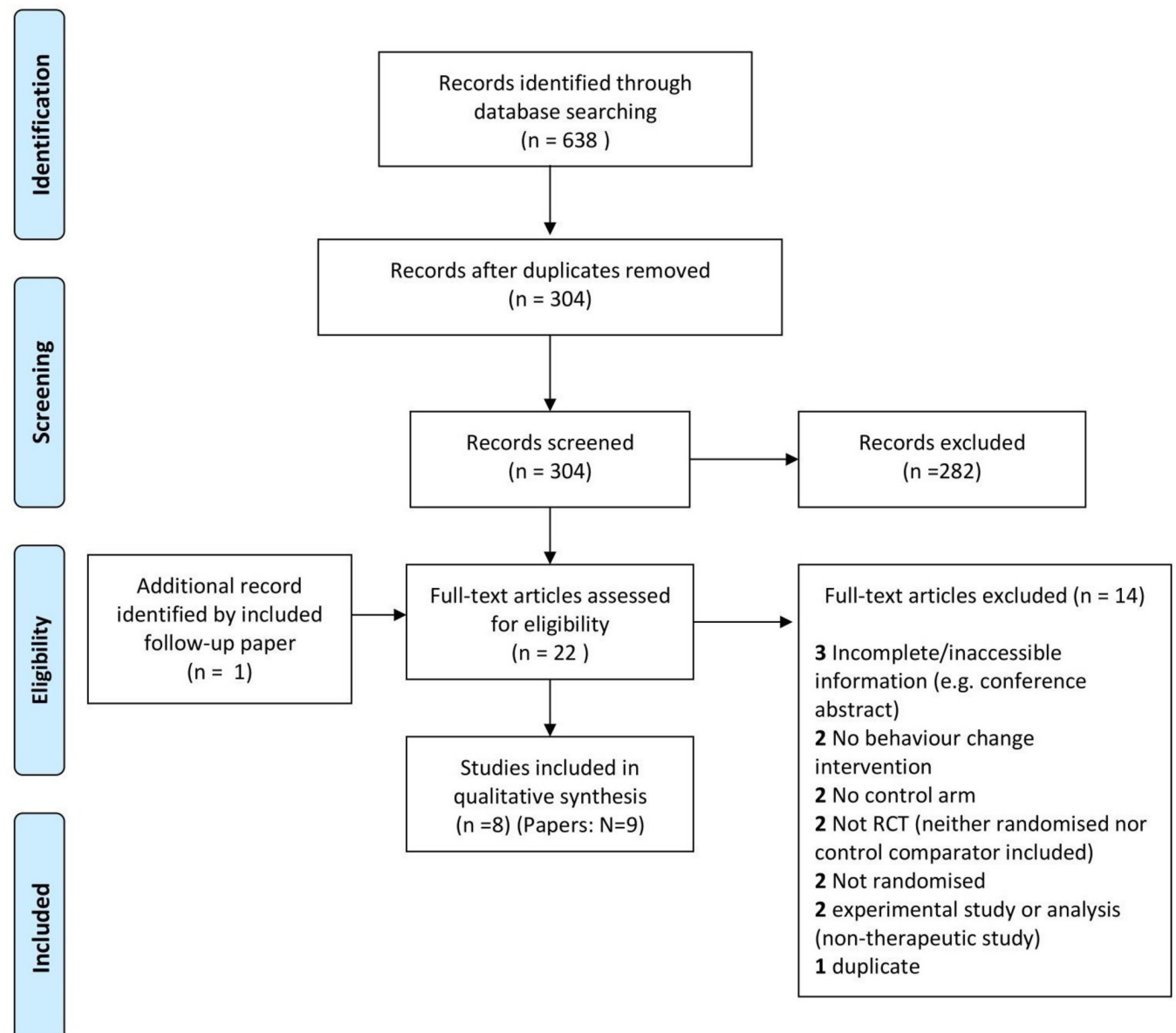

From: Moher D, Liberati A, Tetzlaff J, Altman DG, The PRISMA Group (2009). Preferred Reporting Items for Systematic Reviews and MetaAnalyses: The PRISMA Statement. PLoS Med 6(7): e1000097. doi:10.1371/journal.pmed1000097

Figure 1 PRISMA flow chart. Behaviour change interventions for the management of Raynaud's phenomenon. PRISMA, Preferred Reporting Items for Systematic Reviews and Meta-Analyses; RCT, randomised controlled trial.

unavailable electronically/online therefore not identified within the original search. No additional studies were added as a result of grey or reference list searches. Consultation and resolution with the third reviewer (CE) was required on review of three separate papers. The PRISMA flow chart is given in figure 1.

\section{Study characteristics}

Six of eight studies included participants diagnosed with primary RP; the remaining two used secondary RP samples (secondary to SSc). ${ }^{20} 21$ Studies did not mix participants with primary and secondary RP.

\section{Active treatment conditions}

All studies randomised participants to an active treatment arm and at least one comparator arm. Three studies described one comparator treatment. ${ }^{22-24}$ Five reported two comparator arms. ${ }^{20} 21{ }^{25-27}$ Behaviour change interventions used across the trials included biofeedback $(\mathrm{n}=7)$ and a behavioural intervention $(n=1)$. Three other active treatments formed part of the treatment trials: deep oscillation ${ }^{21}$; autogenic relaxation either alone as an active comparator treatment ${ }^{2025}$ or combined with biofeedback ${ }^{23}$; and Nifedipine, a calcium channel blocker pharmacological intervention in tablet form $(n=1)$ (see interventions section for detail). Only two studies used a no treatment condition as a control comparator. ${ }^{2126}$

\section{Control comparator condition}

Two studies used a placebo as the comparator arm. ${ }^{2427}$ Five studies used interventions that were designed to compensate for the placebo effect or confounding effects of the active treatment arm: gymnastic hand exercises with similar levels of hand movement to performing biofeedback ${ }^{21}$; frontalis electromyograph to counterbalance effects of receiving physiological feedback from biofeedback ${ }^{20} 25$; and autogenic relaxation where the active treatment arm was autogenic relaxation plus biofeedback. ${ }^{23}$ 


\section{Sample characteristics}

Sample sizes ranged from 12 to 313, with a median of 29 (IQR 12). A total of 495 participants were included across studies: 156 in active treatment and 339 in comparator arms.

Females accounted for $73 \%$ of the overall sample. Five studies reported age ranges from 17 years to 65 years. Mean age was reported in four studies, ranging from 28 to 54.4, with a calculated median of 39.5 (IQR 18.7). Ethnicity was not consistently reported and could not be meaningfully estimated. The publication dates of the nine papers (eight studies) ranged from 1978 to 2002, with five of eight studies completed prior to 1984 and none of the included studies published after 2002. One study reported a 12-month follow-up of their original sample $(1981)^{24}$ in a much later paper published in English (1996). ${ }^{28}$ The majority of the studies were USA based and written in the English language $(n=6)$, with one German language and one Swedish language study.

\section{Study outcomes}

All studies targeting primary RP used diary-based approaches to assessing RP (predecessors of the RCS diary), including measures of severity/impact frequency, duration and severity of episodes, with some studies expanding further to include episodes antecedents (ie, perceived triggers or events immediately prior to the RP episode) and a description of the RP episode including symptoms (see table 1). Other outcome measures included physiological measures, physician rated measures, stress and general health measures. One study used the RP VAS subscale of the SHAQ which was developed specifically for the secondary RP population. ${ }^{17}$ Primary outcomes of interest are highlighted in bold.

\section{Interventions}

Seven studies tested 'biofeedback', and one tested 'behavioural treatment' as the active behaviour change interventions for RP.

Biological feedback, or 'biofeedback', interventions in RP are based on the notion that participants can be trained to voluntarily increase blood flow to the extremities based on biological temperature feedback. Biofeedback interventions were similar in procedure across studies (notwithstanding differences in duration/ frequency). An overview of the procedural approach can be summarised herewith: following laboratory assessment, participants were invited to voluntarily increase their skin temperature unaided. Participants were then trained to increase skin temperature based on individualised biological feedback using physiological skin monitoring apparatus, changes in skin temperature were indicated through the use of acoustic sounds which increased in volume along with escalation in skin temperature 20222526 ; visual representations of changes in skin temperature such as a digital panel ${ }^{2326} 27$ or a moving light. ${ }^{22}$ Participants were instructed to increase skin temperature based on this audio/visual feedback. Following completion of training, participants were invited to voluntarily increase finger temperature without the aid of biofeedback. One study did not describe the biofeedback intervention. ${ }^{21}$ The "behavioural intervention,24 28 reported a classical conditioning intervention directed at 'weakening of the unconditioned link between cold and peripheral vasospasms' $\left(\right.$ p. $\left.111^{24}\right)$. Subjects submerged both hands in water that was either $+23^{\circ} \mathrm{C}$ (placebo group) or $+43^{\circ} \mathrm{C}$ (active treatment intervention) and were instructed that the addition of a coloured substance to the water was an active treatment (placebo). Participants were informed that the warmer the water. the better the penetration of the proposed 'active' (placebo) drug, seeking to weaken the link between cold temperatures and vasospasm in the active group. Expectation of treatment effect and self-monitoring were then measured.

One study ${ }^{25}$ delivered 'cognitive stress management' to $50 \%$ of each of the four groups in the participant sample (total $n=32$ ). This intervention consisted of reviewing symptoms, precipitants of episodes, cognitions during episodes and strategies employed to manage episodes. Other active comparators included deep oscillation which targetted restoration of blood supply via an intermittent electrostatic field ${ }^{21}$; autogenic therapy to enhance blood flow through muscle relaxation ${ }^{2025}$; and calcium channel blocker nifedipine ${ }^{27}$ to target pharmacological vasodilation in RP. Of note, no RCTs within this study (or outwith) have assessed the self-management approaches that form recommended first-line intervention outlined in National Institute for Health and Care Excellence (NICE) guidance for the management of $\mathrm{RP}^{10}$ (eg, stress reduction, increased exercise and measures to retain warmth).

Six of eight studies used cold stress tests prior to and/ or following intervention for purposes of maximising demonstration of treatment effect. Temperatures were manipulated within a range of $4-20^{\circ} \mathrm{C}$, for periods of between $7 \mathrm{~min}$ and $17 \mathrm{~min}$ (not all studies specified exact duration).

Treatment interventions varied in length and duration of session; number of sessions ranged from 10-20 sessions (median=12), from $8 \mathrm{~min}$ to $60 \mathrm{~min}$ duration per session (median=40 min). Two trials did not specify treatment session duration. Sessions took place over 2-10 weeks (plus follow-up) on a biweekly, thrice weekly (or unclear) basis. Six studies provided sufficient detail to calculate dose of intervention: biofeedback intervention $(\mathrm{n}=5)$ treatment dose ranged from $375 \mathrm{~min}$ to $1200 \mathrm{~min}$ (median $=420 \mathrm{~min}$ ), and dose for the behavioural intervention $(\mathrm{n}=1)$ was $80 \mathrm{~min}$.

\section{Quality of psychotherapeutic intervention}

Four studies made explicit reference to an underpinning theoretical model of the active treatment approach, providing rationale for the application of biofeedback $(n=3)^{2325} 27$ and behavioural theory $(n=1)^{24} 28$ in RP. The remaining four studies reported the application of biofeedback but in the absence of theoretical explanation as to the relevance of application in RP. Studies referred 


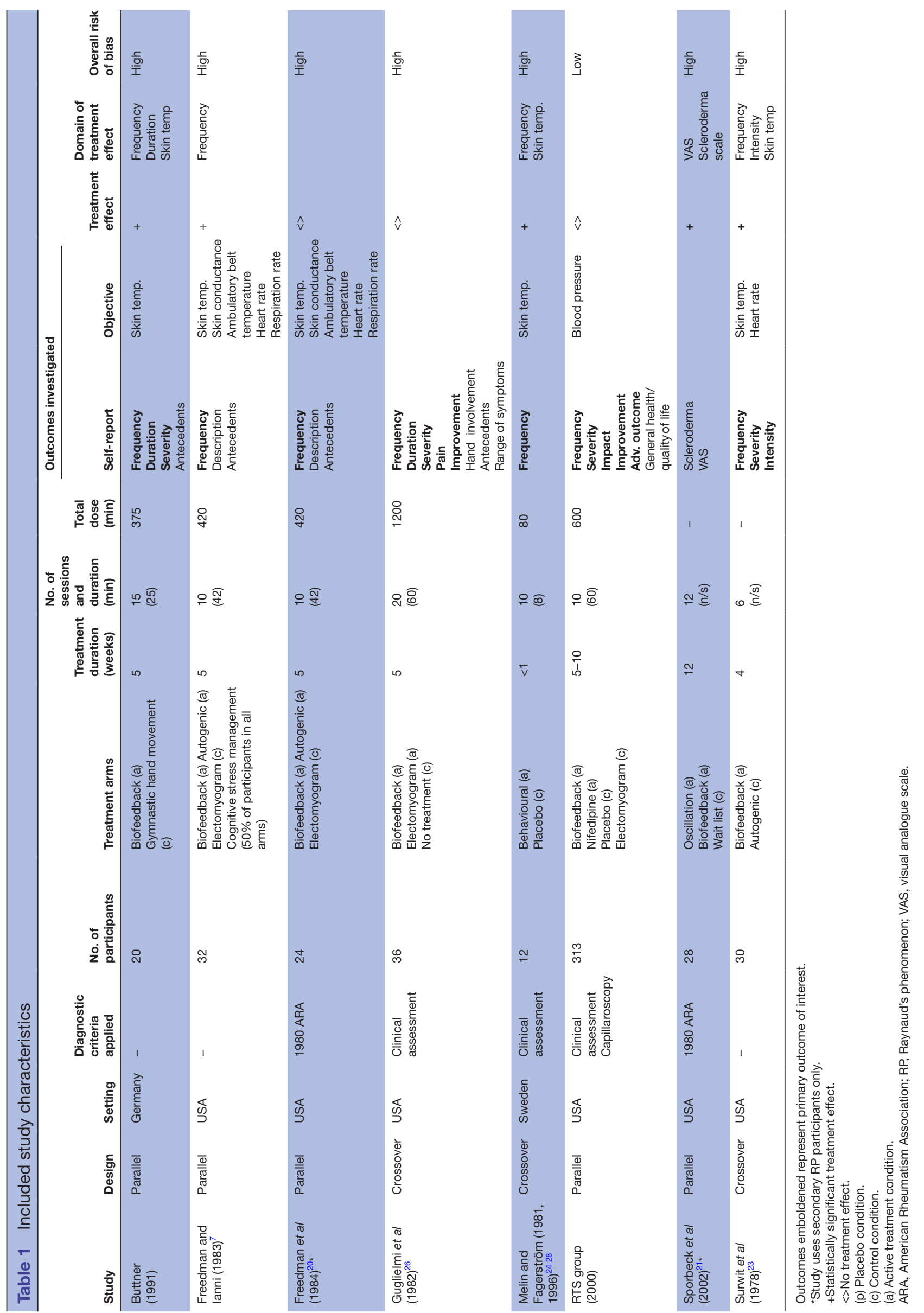

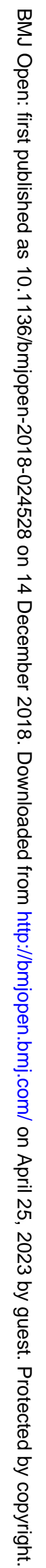


Table 2 Risk of bias assessment

\begin{tabular}{|c|c|c|c|c|c|c|c|}
\hline & $\begin{array}{l}\text { Random } \\
\text { sequence } \\
\text { generation }\end{array}$ & $\begin{array}{l}\text { Allocation } \\
\text { concealment }\end{array}$ & $\begin{array}{l}\text { Blinding of } \\
\text { participants }\end{array}$ & $\begin{array}{l}\text { Blinding of } \\
\text { assessors }\end{array}$ & $\begin{array}{l}\text { Incomplete } \\
\text { outcome } \\
\text { reporting }\end{array}$ & $\begin{array}{l}\text { Selective } \\
\text { outcome } \\
\text { reporting }\end{array}$ & Other bias \\
\hline Buttner et $\left.a\right|^{22}$ & $?$ & $?$ & $?$ & + & - & + & + \\
\hline Freedman and Ianni ${ }^{7}$ & $?$ & $?$ & + & $?$ & + & + & + \\
\hline Freedman et $a l^{20}$ & $?$ & $?$ & + & + & + & + & + \\
\hline Guglielmi et $a l^{26}$ & + & + & - & - & - & + & - \\
\hline $\begin{array}{l}\text { Melin and Fagerström } \\
(1981 / 1996)^{24}\end{array}$ & $?$ & $?$ & $?$ & + & + & - & - \\
\hline RTS group (2000) & - & - & + & - & - & + & $?$ \\
\hline Sporbeck et $a l^{21}$ & $?$ & $?$ & + & $?$ & $?$ & - & + \\
\hline Surwit et $\left.a\right|^{23}$ & $?$ & $?$ & + & $?$ & + & + & + \\
\hline
\end{tabular}

?=unsure.

$+=$ high risk.

$-=$ low risk of bias.

RTS, Raynaud's Treatment Study.

to earlier work on the use of biofeedback, looking to replicate or improve previous studies. The underpinning theory relating to 'cognitive stress management' was referred to in the main report of a biofeedback for RP study, ${ }^{25}$ with indications that a protocol had been adhered to and made available on request; however, authors note that the analysis of the data is limited by small cell size.

One study reported the level of therapist training ${ }^{26}$ describing those facilitating the intervention as 'assistants'; however, no studies reported level of therapist training or any additional information pertaining to qualifications of therapists delivering the interventions. Finally, despite all studies bearing an RCT design, no studies reported checking treatment integrity/fidelity of interventions.

\section{Risk of bias}

Risk of bias assessments for each of the studies is shown in table 2. Inter-rater reliability between JD and JP was $73 \%$ $(\mathrm{k}=0.59)$, indicating fair agreement. ${ }^{29}$ The main discrepancy between raters was inconsistent interpretation of selective reporting and lack of agreement in four of seven domains in one paper. ${ }^{26}$ This was attributed to the variability in quality and completeness of reporting. The final risk of bias assessment includes the adjudicated ratings (table 2).

As highlighted in the risk of bias assessment table (table 2), a lack of clarity in reporting random sequence generation, allocation concealment and blinding of participants were common limitations of the studies assessed. Overall risk of bias was assessed based on risk across the seven domains and the following considerations: due to the nature of psychologically based interventions, high risk of bias is common in domains of blinding of participants and assessors and would be regarded accordingly; incomplete and selective outcome reporting were deemed important in relation to the stated second study objective to inform development of future treatment trials, therefore more weight is given to these domains; random sequence generation and allocation of concealment are considered pertinent and particularly important as regards interpreting the comparative efficacy and informing future research; uncertainty in several domains was regarded as susceptibility to high risk of bias and compromising study objectives; and as indicated in the Cochrane handbook, ${ }^{18}$ high risk of bias across most domains is associated with overestimates of effect, which would compromise the study objective to assess relative efficacy. Based on these particular considerations, the majority of the studies rated as high risk of bias.

The lowest overall risk of bias was in the Raynaud's Treatment Study (RTS) group. This was the largest study and benefited from higher quality reporting typical of larger well-controlled RCTs. The Guglielmi study ${ }^{26}$ was a smaller, well-reported but poorly controlled study in relation to random allocation methods and allocation of concealment; the randomisation process involved matching on age, frequency and duration of attacks and was lacking in transparency. All other studies indicated high risk of bias or uncertainty across the majority of domains and were thus considered overall high risk of bias. Two of eight studies included were published as short reports. All authors were contacted for information to support a comprehensive assessment of risk of bias; however, data were no longer available due to the studies having been completed up to 36 years previously or author unresponsiveness.

\section{GRADE}

The quality of the evidence for all seven primary outcomes was judged to be very low. No data could be extracted for analysis meaning that by definition our confidence in judging the efficacy and safety of behaviour change interventions is low and any estimate would be highly likely to change with the addition of new evidence. As such, we 
Table 3 Means and SD of study primary outcomes

\section{Immediately post-treatment}

\begin{tabular}{|c|c|c|c|c|c|}
\hline & \multirow[b]{2}{*}{ N (Post) } & \multicolumn{2}{|c|}{$\begin{array}{l}\text { Number of attacks } \\
\text { (monthly) }\end{array}$} & \multicolumn{2}{|c|}{ Duration of attacks } \\
\hline & & $\mathbf{R x}$ & Control & $\mathbf{R x}$ & Control \\
\hline Freedman and lanni ${ }^{7}$ & 32 & & & & \\
\hline Guglielmi et $a l^{26}$ & 36 & $\begin{array}{l}45 \\
\text { (calculated mean) }\end{array}$ & $\begin{array}{l}50.8 \\
\text { (calculated mean) }\end{array}$ & & \\
\hline Melin and Fagerström²4 & $\begin{array}{l}6 \\
11\end{array}$ & $\begin{array}{l}4.3 \\
1.5\end{array}$ & $\begin{array}{l}2.8 \\
1.2\end{array}$ & & \\
\hline RTS group (2000) & 313 & & & & \\
\hline
\end{tabular}

*Studies using secondary Raynaud's phenomenon participants only. RTS, Raynaud's Treatment Study.

judged that an empty summary of findings table would be unhelpful.

\section{Data synthesis and descriptive (narrative) analysis of findings} Primary and secondary outcomes

Of the seven primary outcomes, there were insufficient data reported on any of the outcomes to assess comparative safety and efficacy. For the primary outcome of RP episode frequency three studies reported means and $\mathrm{SD}^{20} 22$ 24; however, two of three studies used primary RP samples (therefore only two studies could be meaningfully compared) and time points were unclear on one study. ${ }^{20}$ This restricted any further robust comparative measurement of efficacy as regards frequency. Means and/ or SD could not be reliably calculated based on available or acquired data for the remaining studies.

One study reported means and $\mathrm{SD}$ of duration of $R P$ episodes $^{22}$; however, only one other study examined duration but did not provide relevant data. Attempts to recover data directly from authors were unsuccessful.

Of the five remaining primary outcomes (severity/ impact, pain intensity, patient assessment of disability/interference on daily activities, adverse events and withdrawals) data were largely missing with the exception of narrative information relating to adverse events in one study, ${ }^{27}$ and full sample retention indicated in all but two studies that reported an attrition rate of $\leq 10 \% .{ }^{23}{ }^{26}$ The extensive missing and/or incomplete data formed the rationale for the lack of viability of a meta-analysis. Table 3 reports all reported means and SD pertaining to study primary outcomes, highlighting the paucity of reported data.

In relation to predetermined secondary outcomes of interest (physician global assessment of severity/impact of RP; patient global assessment of function/disability secondary to RP; change in digital ulceration; treatment preference and general improvement; and self-reported overall improvement), data available on patient perceived improvement and quality of life on one study ${ }^{27}$ indicated that all treatments had little effect on quality of life; however, both patients and clinicians rated a high degree of improvement in the pharmacological intervention (nifedipine) in comparison with biofeedback and control conditions. The Guglielmi ${ }^{26}$ paper reported findings relating to perceived improvement; however, unplanned post hoc analysis based on learning criteria associated with the biofeedback technique obscured meaningful interpretation of results. In relation to these and other aforementioned secondary outcomes, there was again insufficient data to warrant a meta-analysis. Data pertaining to anxiety/stress were generally measured using unvalidated methods such as heart/respiration rates or other unstandardised measures. Mood was not investigated.

In summary of study reported outcomes, five of the eight studies reported positive outcomes in at least one domain. This consisted of relative reductions in frequency $(\mathrm{n}=3)^{722}{ }^{24}$; finger-tip temperature $(\mathrm{n}=3)^{22-24}$; VAS pain score $(n=1)^{21}$; duration of episodes $(n=1)^{22}$; and intensity of episodes $(\mathrm{n}=1),{ }^{23}$ with three studies demonstrating significant change in more than one area. Three of eight studies reported no difference in any domains. ${ }^{20} 2627$ Six of eight studies reported no viable data in any domains pertaining to primary and secondary outcomes, ${ }^{20} 212325-27$ two of these provided data that required further calculations to produce or estimate mean values; however, the data/calculated means were deemed insufficiently reliable to use in analysis. ${ }^{20} 26$

\section{DISCUSSION}

Our primary objective was to assess the comparative safety and efficacy of a range of behaviour change interventions 
Table 4 Future trial considerations

\section{Trial design reported flaws Future considerations}

Underpinning theory and conceptual framework

Classification and inclusion criteria

\begin{tabular}{|c|c|}
\hline Measurement & $\begin{array}{l}\text { The RCS diary (or any future validated tools for assessing RP) should be employed as a } \\
\text { standardised tool of choice in RP trials to allow for meaningful comparisons across treatment } \\
\text { conditions and studies and gather relevant outcome data in one measure. } \\
\text { Consideration should be given to technologically enhanced methods to increase reliability } \\
\text { such as ecological momentary interventions to allow provision of regular prompts for RCS } \\
\text { completion, rather than over-reliance on self-report measures that may be confounded by } \\
\text { recall bias. } \\
\text { Verification of RP episodes through the use of colour charts (see RTS study) and } \\
\text { capillaroscopy may provide reliable data for use with self-report measures. } \\
\text { Functional assessment of digital microvascular function (eg, laser-derived imaging modalities } \\
\text { or thermal imaging to assess digital vascular function) should be used as exploratory } \\
\text { endpoints to triangulate with subjective measures, although further validation of non-invasive } \\
\text { microvascular imaging techniques is necessary before they can be fully incorporated into the } \\
\text { endpoint model of RP clinical trials. }{ }^{52-54} \\
\text { Patient-reported outcome instruments and objective imaging modalities could be applied as } \\
\text { coprimary endpoints in future clinical trials. } \\
\text { Measures relating to psychological well-being (eg, quality of life, anxiety/stress and pain) } \\
\text { should be used due to the known pivotal role of these factors in self-management and } \\
\text { outcome. }\end{array}$ \\
\hline Treatment arms and sample & $\begin{array}{l}\text { Appropriately powered samples with full reporting of findings will generate more reliable } \\
\text { results. }\end{array}$ \\
\hline Protocol/procedure reporting & $\begin{array}{l}\text { The Template for Intervention Description and Replication }\left(T_{I D i e R}{ }^{40}\right) \text { should be adopted for } \\
\text { the reporting of behaviour change interventions in RP. This would provide consistency and } \\
\text { transparency in reporting, making relevant information available for scrutiny. TiDieR requires } \\
\text { information relating to the experience and training required for the intervention, providing } \\
\text { further clarity on the necessary skills to deliver the intervention. } \\
\text { Integrity to a protocol-driven intervention procedure with appropriate quality control } \\
\text { measures such as (A) fidelity checks and (B) clarity of reporting for risk of bias and purposes } \\
\text { of replication. This is likely to increase compliance and improve the quality and outcomes of } \\
\text { treatment interventions. }\end{array}$ \\
\hline
\end{tabular}

- The treatment model or underpinning mechanisms of the intervention should be clearly stated to provide a clear rationale and transparency relating the scientific credibility of the intervention. The intervention mapping framework ${ }^{51}$ provides a good example of a mapping tool used in the development of behaviour change interventions and would be highly relevant to treatment development in RP.

- Standardised use of ARA diagnostic criteria for inclusion criteria will allow clearer comparison of outcomes and reduce risk of bias.

- Systematic use of a diagnostic criteria will introduce a higher degree of objectivity in assessment.

- Due to the widely acknowledged disparity in the known underlying pathogenesis between primary and secondary RP, these groups should be considered distinct and separate. standardised tool of choice in RP trials to allow for meaningful comparisons across treatment

Consideration should be given to technologically enhanced methods to increase reliability

Verification of RP episodes through the use of colour charts (see RTS study) and or thermal imaging to assess digital vascular function) should be used as exploratory endpoints to triangulate with subjective measures, although further validation of non-invasive microvascular imaging techniques is necessary before they can be fully incorporated into the endpoint model of RP clinical trials. ${ }^{52-54}$ coprimary endpoints in future clinical trials.

Measures relating to psychological well-being (eg, quality of life, anxiety/stress and pain) should be used due to the known pivotal role of these factors in self-management and outcome.

Appropriately powered samples with full reporting of findings will generate more reliable the reporting of behaviour change interventions in RP. This would provide consistency and (1) further clarity on the necessary skills to deliver the intervention. measures such as (A) fidelity checks and (B) clarity of reporting for risk of bias and purposes of replication. This is likely to increase compliance and improve the quality and outcomes of

A no-treatment/wait list control or equivalent should be adopted in future trials as a minimum parator.

controlled for as a covariate where possible, measured and reported on

counterbalance bias-attributed difficulties achieving participant blinding in behaviour change and other similar therapeutic modalities. for the management of symptoms associated with primary and secondary RP. Due to limitations in study design, reporting and the absence of meaningful data, we are unable to offer any effect estimates. Essentially, there is no evidence to support or refute claims made for the efficacy and safety of behaviour change interventions for the management of RP.
Given the burden of RP, ${ }^{2} 103031$ the relatively mature development of psychological interventions in cognate fields (eg, chronic pain ${ }^{32}$ ) and potential for self-management interventions given the importance of cold exposure and stress as exacerbating factors for RP, it is surprising that there is no modern tradition of therapy development and that little work has been undertaken 
in this field in the last 20 years. We have speculated elsewhere that this is due to the deleterious effect of the RTS study. ${ }^{31}$ The RTS was a high-profile negative study conducted and reported in Archives of Internal Medicine which, from a modern perspective, used a treatment modality waning medical support owing to absent or unimpressive outcomes. Advancement in the application of biofeedback appears to have ceased at the point at which a high-quality study deemed it ineffective.

The question as to the efficacy and safety of behaviour change interventions remains unanswered, and the case for further investigation persists if we are to continue championing the utilisation of self-management approaches in the management of RP, as per NICE guidance. ${ }^{10}$ We have drawn lessons from the existing data in relation to study design, conduct and reporting. Comparison has been most notably limited by heterogeneity in study design and measurement. There is a need for further development in a programme of research in behavioural influences in the onset and management of RP episodes (eg, stress/anxiety, behavioural change including retaining more warmth increasing exercise), which are central to the primary and secondary prevention of RP episodes. With up to a third of RP episodes stress/anxiety related ${ }^{25}$ and the remainder associated with cold exposure, episodes are potentially preventable. Due to the pivotal role of psychological and physical stress as a trigger in RP, psychophysiology in RP appears to be important: the essential role of the limbic hypothalamic-pituitary-adrenal axis and neuroendocrine system in stress and physiological disregulation has been explored elsewhere in medical conditions complicated by anxiety, ${ }^{34-36}$ however, not within RP.

The cognitive-affective perspective of RP is unchartered territory, despite the known reciprocal negative impact of stress and anxiety on the body and the role of anxiety/ stress in RP. Studies report significant associations between anxiety and increases in both severity and pain in RP, ${ }^{37}$ and despite clear evidence for the effective treatment of anxiety and pain in other long-term conditions, ${ }^{38}{ }^{39}$ there are no recommended non-pharmacological interventions for $\mathrm{RP}^{104041}$ and no known evidence to support efficacy of behavioural and lifestyle changes recommended by NICE. ${ }^{10}{ }^{41}$ There has been no research in RP examining illness beliefs, psychological distress, knowledge deficits or other non-medical factors commonly associated with outcome. Related conditions such as rheumatoid arthritis have reported improved outcomes through illness belief targeted behaviour change interventions ${ }^{42}$ with emerging evidence for the efficacy of behavioural and lifestyle behaviour change interventions in other health conditions ${ }^{43}$ but not in RP, despite recommendations. ${ }^{10}$ These factors could serve as a target for intervention in $\mathrm{RP}^{44}$ The complex interplay between cognitive, social and behavioural factors that underpin a stress response warrant further investigation in RP.

The findings of this systematic review are limited to the inclusion of RCTs only. The application of these stringent criteria is likely to have produced fewer results than a broader inclusion of uncontrolled studies. However, RP clinical trials are particularly prone to placebo effects, ${ }^{14}$ and interpretation of open-label studies of RP is challenging. We do not believe that the inclusion of non-randomised studies would have reduced any uncertainty over efficacy and safety. We also note the moderate agreement on risk of bias between raters ( $\mathrm{k}=0.59,73 \%$ agreement). The lack of agreement centred on one specific paper ${ }^{26}$ and poor agreement on the 'selective reporting' domain, which was adjudicated by a third author (CE). We suggest that the broad heterogenity of the data and study design obscured reporting and, in places, the assessment of reporting.

With regard to specific trial design flaws and future considerations within the field of RP treatment, there were four features of trial design and reporting that hampered any analysis of efficacy and safety of behaviour change interventions in this review. First, inadequate measurement, storage and/or reporting of data meant no meta-analysis was possible. Second, the historical lack of consensus over nosology, measurement and classification led inevitably to a lack of clarity over exactly who was entering trials. Third, smaller sample trials even when properly reported threaten precision of the effect estimates and introduce the possibility of unreliability; while smaller samples are not problematic per se, the majority of the studies included also demonstrate threats to reliability such as the absence of robust assessment measures and moderate to high risk of bias. Finally, there were multiple considerations throughout the individual studies about the content, dose, conduct and delivery of the therapies, ranging from two studies that discussed the potential implications of change in weather over the course of treatment, ${ }^{22}{ }^{26}$ difficulties in the acquisition and application of training skills, ${ }^{26}{ }^{27}$ and the validity and generalisability of biofeedback assessed via finger-tip skin temperature. ${ }^{202324}$ These considerations may be historical given dates of publication range from 1978 to 2002. This predates the development and subsequent endorsement of outcome measures such as the RCS diary disease classification criteria and clinical trial reporting standards. The RCS diary is currently the preferred outcome measure for scleroderma-related RP clinical trials and has been endorsed by the Scleroderma Clinical Trials Consortium ${ }^{45}$ and Outcome Measures in Rheumatoid Arthritis Clinical Trials (OMERACT) ${ }^{46}$ While not formally validated for primary RP, it has been successfully used in studies of mixed populations of patients. ${ }^{47}$ Recent work has highlighted limitations to the RCS diary and work is underway to develop novel patient-reported outcome instruments that might complement diarybased approaches in future clinical trials assessing the outcome of behaviour intervention on the severity and impact of RP. ${ }^{4-50}$

A summary of the recommended critical features of any new trials in this field is presented (table 4). 


\section{CONCLUSION}

This is the first systematic review of behaviour change interventions for the management of primary and secondary RP. The study produced no evidence to support or refute claims for the efficacy and safety of behaviour change interventions for the management of RP. Little work has focused on behaviour change in RP management in recent years despite the importance attached to self-management in clinical practice guidelines. ${ }^{910}$ There remains a strong case for developing and testing behavioural-based interventions that focus the self-management of RP by addressing (A) behavioural avoidance of environmental exposure to triggers of RP attacks, (B) promoting a cognitive-affective understanding of RP and (C) learning from the vast body of evidence underpinning behaviour change in complex, poorly understood medical conditions that are amenable to intervention.

\section{ETHICS AND DISSEMINATION}

This systematic review did not require ethical approval because it summarises published studies with non-identifiable data. This systematic review is reported according to the PRISMA guidelines (see online supplementary materials, table 1).

Acknowledgements Elizabeth Sheils for her assistance with the manuscript and Justin Hodds for guidance on defining search terms.

Contributors All authors were involved in developing the focus of the systematic review, arriving at the final conception of the proposed systematic review through an iterative process. JD produced the first draft of the paper and supplementary information, coordinated and assimilated comments from JP and CE, approved the final version for publication and is the guarantor of the manuscript. JP also critically revised successive drafts of the manuscript, provided expert advice and intellectual input regarding the subject area (Raynaud's phenomenon) and approved the final version for publication. CE critically revised successive drafts of the manuscript, provided expert advice on systematic review methodology and approved the final version for publication.

Funding The authors have not declared a specific grant for this research from any funding agency in the public, commercial or not-for-profit sectors.

Competing interests None declared.

Patient consent Not required.

Provenance and peer review Not commissioned; externally peer reviewed.

Data sharing statement The data extraction form, search terms and other supplementary materials defined a priori are available for open access at http:// bmjopen.bmj.com/content/7/8/e017039. Published papers form the 'data' of the present study and can be acquired from the corresponding author on request; the majority are available online.

Open access This is an open access article distributed in accordance with the Creative Commons Attribution Non Commercial (CC BY-NC 4.0) license, which permits others to distribute, remix, adapt, build upon this work non-commercially, and license their derivative works on different terms, provided the original work is properly cited, appropriate credit is given, any changes made indicated, and the use is non-commercial. See: http://creativecommons.org/licenses/by-nc/4.0/.

\section{REFERENCES}

1. Johnson JM, Kellogg DL. Local thermal control of the human cutaneous circulation. J Appl Physiol 2010;109:1229-38.

2. Pauling JD, Domsic RT, Saketkoo LA, et al. A multi-national qualitative research study exploring the patient experience of
Raynaud's phenomenon in systemic sclerosis. Arthritis Care Res 2017.

3. Pauling JD, Saketkoo LA, Matucci Cerinic M, et al. The patient experience of Raynaud's phenomenon in systemic sclerosis. Rheumatology. In Press.;2018.

4. Denton CP, Khanna D. Systemic sclerosis. Lancet 2017;390:1685-99.

5. Mayes MD, Lacey JV, Beebe-Dimmer J, et al. Prevalence, incidence, survival, and disease characteristics of systemic sclerosis in a large US population. Arthritis Rheum 2003;48:2246-55.

6. Watson HR, Robb R, Belcher G, et al. Seasonal variation of Raynaud's phenomenon secondary to systemic sclerosis. J Rheumatol 1999;26:1734-7.

7. Freedman RR, lanni P. Role of cold and emotional stress in Raynaud's disease and scleroderma. Br Med J 1983;287:1499-502.

8. Freedman RR, lanni P. Effects of general and thematically relevant stressors in Raynaud's disease. J Psychosom Res 1985;29:275-80.

9. Hughes M, Ong VH, Anderson ME, et al. Consensus best practice pathway of the UK Scleroderma Study Group: digital vasculopathy in systemic sclerosis. Rheumatology 2015;54:2015-24.

10. NICE. NIfCaHE. Raynaud's phenomenon: clinical knowledge summary. 2014.

11. Vermeire $\mathrm{E}$, Hearnshaw $\mathrm{H}$, Van Royen $\mathrm{P}$, et al. Patient adherence to treatment: three decades of research. A comprehensive review. J Clin Pharm Ther 2001;26:331-42.

12. Daniels J, Pauling JD, Eccelston C. Behaviour change interventions for the management of Raynaud's phenomenon: a systematic review protocol. BMJ Open 2017;7:e017039.

13. Chambless DL, Hollon SD. Defining empirically supported therapies. J Consult Clin Psychol 1998;66:7-18.

14. Gladue $\mathrm{H}$, Maranian $\mathrm{P}$, Paulus HE, et al. Evaluation of test characteristics for outcome measures used in Raynaud's phenomenon clinical trials. Arthritis Care Res 2013;65:630-6.

15. Pope J, Rirash F, Tingey PC, et al. Drug interventions versus placebo for the treatment of Raynaud's phenomenon: generic protocol. Cochrane Database Syst Rev 2015;350.

16. Wigley FM, Korn JH, Csuka ME, et al. Oral iloprost treatment in patients with Raynaud's phenomenon secondary to systemic sclerosis: a multicenter, placebo-controlled, double-blind study. Arthritis Rheum 1998;41:670-7.

17. Steen VD, Medsger TA. The value of the health assessment questionnaire and special patient-generated scales to demonstrate change in systemic sclerosis patients over time. Arthritis Rheum 1997;40:1984-91.

18. In: Higgins JP, Green S, eds. Cochrane handbook for systematic reviews of interventions: John Wiley \& Sons, 2011.

19. Guyatt GH, Oxman AD, Vist GE, et al. GRADE: an emerging consensus on rating quality of evidence and strength of recommendations. BMJ (Clinical research ed. 2008;336:924-6.

20. Freedman RR, lanni P, Wenig P. Behavioral treatment of Raynaud's phenomenon in scleroderma. J Behav Med 1984;7:343-53.

21. Sporbeck B, Mathiske-Schmidt K, Jahr S, et al. Effect of Biofeedback and deep oscillation on Raynaud's Phenomenon secondary to systemic sclerosis: results of a controlled prospective randomized clinical trial. Rheumatol Int 2002;32:14.

22. Buttner M, Henschel G, Spengel FA. The efficacy of temperature biofeedback in the treatment of Raynaud's disease. Vasa - Journal of Vascular Diseases 1991;20:411-5.

23. Surwit RS, Pilon RN, Fenton $\mathrm{CH}$. Behavioral treatment of Raynaud's disease. J Behav Med 1978;1:323-35.

24. Melin B, Fagerström K-O. Behandling av perifer vasospasm. Cogn Behav Ther 1981;10:97-111.

25. Freedman RR, lanni $P$, Wenig P. Behavioral treatment of Raynaud's disease. J Consult Clin Psychol 1983;51:539-49.

26. Guglielmi RS, Roberts AH, Patterson R. Skin temperature biofeedback for Raynaud's disease: a double-blind study. Biofeedback Self Regul 1982;7:99-120.

27. Raynaud's treatment study investigators. Comparison of sustained-release nifedipine and temperature biofeedback for treatment of primary raynaud phenomenon. Arch Intern Med 2000;160:1101-8.

28. Melin B, Fagerström K-O. A one year follow-up of a placebo controlled behavioural treatment of peripheral vasospasm. Cogn Behav Ther 1996;25:27-35.

29. McHugh ML. Interrater reliability: the kappa statistic. Biochem Med 2012;22:276-82.

30. Maundrell A, Proudman SM. Raynaud's Phenomenon. In: Epidemiology of Raynaud's phenomenon. New York: Springer, 2015:21-35.

31. Eccleston C. Embodied: the psychology of physical sensation: Oxford University Press, 2016. 
32. Williams AC, Eccleston C, Morley S. Psychological therapies for the management of chronic pain (excluding headache) in adults. Cochrane Libr 2012;11:CD007407.

33. Hewlett S, Ambler N, Almeida C, et al. Protocol for a randomised controlled trial for Reducing Arthritis Fatigue by clinical Teams (RAFT) using cognitive-behavioural approaches. BMJ Open 2015;5:e009061.

34. Daniels J, Turner-Cobb JM. Adjuvant psychological therapy in longterm endocrine conditions. Clin Endocrinol 2017;86:772-7.

35. Daniels J, Sheils E. A complex interplay: cognitive behavioural therapy for severe health anxiety in addison's disease to reduce emergency department admissions. Behav Cogn Psychother 2017;45:419-26.

36. Straub RH, Dhabhar FS, Bijlsma JW, et al. How psychological stress via hormones and nerve fibers may exacerbate rheumatoid arthritis. Arthritis Rheum 2005;52:16-26.

37. Brown KM, Middaugh SJ, Haythornthwaite JA, et al. The effects of stress, anxiety, and outdoor temperature on the frequency and severity of Raynaud's attacks: the Raynaud's Treatment Study. J Behav Med 2001;24:137-53.

38. Olatunji BO, Cisler JM, Deacon BJ. Efficacy of cognitive behavioral therapy for anxiety disorders: a review of meta-analytic findings. Psychiatr Clin North Am 2010;33:557-77.

39. Tyrer P, Cooper S, Salkovskis $\mathrm{P}$, et al. Clinical and cost-effectiveness of cognitive behaviour therapy for health anxiety in medical patients: a multicentre randomised controlled trial. The Lancet 2014;383:219-25.

40. Ennis H, Hughes M, Anderson ME, et al. Calcium channel blockers for primary Raynaud's phenomenon. Cochrane Database Syst Rev 2016;2:CD002069.

41. Pope JE. Raynaud's phenomenon (primary). BMJ Clin Evid 2011;2011.

42. Baum A, Revenson TA, Singer JE, eds. Handbook of Health Psychology: Psychology press, 2012.

43. Cramp F, Berry J, Gardiner M, et al. Health behaviour change interventions for the promotion of physical activity in rheumatoid arthritis: a systematic review. Musculoskeletal Care 2013;11:238-47.

44. WHO. Adherence to long-term therapies. 2013. http://apps.who.int/ medicinedocs/en/d/Js4883e/9.1.3.html (Accessed 20th Oct 2017).
45. Khanna D, Lovell DJ, Giannini E, et al. Scleroderma Clinical Trials Consortium. Development of a provisional core set of response measures for clinical trials of systemic sclerosis. co-authors. Ann Rheum Dis 2008:67:703-9.

46. Merkel PA, Clements PJ, Reveille JD, et al. Current status of outcome measure development for clinical trials in systemic sclerosis. Report from OMERACT 6. J Rheumatol 2003;30:1630-47.

47. Chung L, Shapiro L, Fiorentino D, et al. MQX-503, a novel formulation of nitroglycerin, improves the severity of Raynaud's phenomenon: a randomized, controlled trial. Arthritis Rheum 2009;60:870-7.

48. Baron M, Kahaleh B, Bernstein EJ, et al. An interim report of the scleroderma clinical trials consortium working groups. J Scleroderma Relat 2018.

49. Pauling JD, Frech TM, Hughes M, et al. Patient-reported outcome instruments for assessing Raynaud's phenomenon in systemic sclerosis: A SCTC vascular working group report. J Scleroderma Relat Disord 2018;3:249-52.

50. Pauling JD, Saketkoo LA, Domsic RT. Patient Perceptions of the Raynaud's condition score diary provide insight into its performance in clinical trials of Raynaud's phenomenon: comment on the article by Denton et al. Arthritis Rheumatol 2018;70:973-4.

51. Bartholomew LK, Parcel GS, Kok G. Intervention mapping: a process for developing theory- and evidence-based health education programs. Health Educ Behav 1998;25:545-63.

52. Wilkinson JD, Leggett SA, Marjanovic EJ, et al. A multicenter study of the validity and reliability of responses to hand cold challenge as measured by laser speckle contrast imaging and thermography: outcome measures for systemic sclerosis-related Raynaud's Phenomenon. Arthritis Rheumatol 2018;70:903-11.

53. Pauling JD, Shipley JA, Harris ND, et al. Use of infrared thermography as an endpoint in therapeutic trials of Raynaud's phenomenon and systemic sclerosis. Clin Exp Rheumatol 2012;30:S103-15.

54. Pauling JD, Shipley JA, Hart DJ, et al. Use of laser speckle contrast imaging to assess digital microvascular function in primary raynaud phenomenon and systemic sclerosis: a comparison using the raynaud condition score diary. J Rheumatol 2015;42:1163-8.

55. Zelen M. A new design for randomized clinical trials. N Engl J Med 1979;300:1242-5. 